\title{
Ocho décadas de evolución en salud pública desde el Instituto de Diagnóstico y Referencia Epidemiológicos
}

\author{
José Alberto Díaz-Quiñonez
}

Instituto de Diagnóstico y Referencia Epidemiológicos "Dr. Manuel Martínez Báez", Secretaría de Salud, Ciudad de México, México

Despertar a la historia significa adquirir conciencia de nuestra singularidad, momento de reposo reflexivo antes de entregarnos al hacer.

OCTAVIO PAZ

El Instituto de Salubridad y Enfermedades Tropicales (ISET) inició actividades a principios de $1938^{*, 2}$ y fue formalmente inaugurado el 18 de marzo de 1939, en conmemoración del primer aniversario de la expropiación petrolera. ${ }^{3}$ En el ISET se realizaron estudios científicos para atender las epidemias y endemias causadas por patologías tropicales, exóticas 0 las agrupadas en la llamada "medicina colonial". Durante cinco décadas, el ISET integró una gran cantidad de equipos interdisciplinarios de investigación en salud, contribuyó a la profesionalización de notables generaciones de sanitaristas mexicanos y obtuvo merecido prestigio internacional.

Con esta sólida y prestigiosa tradición, en el quincuagésimo aniversario de su inauguración, el ISET se convirtió en el Instituto Nacional de Diagnóstico y Referencia Epidemiológicos "Dr. Manuel Martínez Báez". Esta nueva etapa, de poco más de una década, se caracterizó por la contribución del Instituto en encuestas seroepidemiológicas nacionales ${ }^{4}$ y el enorme impulso que recibió la investigación. ${ }^{5,6}$ La promulgación de la Ley de los Institutos Nacionales de Salud durante la primera transición democrática en el año 2000, que tuvo por objetivo regular su organización y funcionamiento, fomentar la investigación, la enseñanza y la prestación de servicios, formalizó la siguiente evolución del Instituto. ${ }^{7}$

* Ramírez-Hernández JA, Guzmán-Bracho C, Díaz-Quiñonez JA. Desde el ISET al InDRE. I. Instituto de Salubridad y Enfermedades Tropicales: Génesis y primeros años, 19341940. Gac Med Mexico. 2019 (en prensa).
En los últimos 20 años, los cambios han sido vertiginosos. El mundo se transformó ante la amenaza del terrorismo internacional en septiembre de 2001 y el concepto de riesgo biológico asumió otro significado para los laboratorios de salud pública. El sistema nacional de vigilancia epidemiológica incorporó la vigilancia del bioterrorismo y el Instituto implementó procedimientos diagnósticos confirmatorios para el ántrax y otras enfermedades emergentes. ${ }^{8}$ Se iniciaron los esfuerzos por implementar un sistema institucional de garantía de calidad ante la ineluctable participación en las redes regionales y globales recién constituidas. El establecimiento de la Iniciativa Global para la Seguridad en Salud permitió la participación del Instituto en foros y discusiones del más alto nivel. Durante la pandemia de influenza en 2009 se estableció el algoritmo diagnóstico y una red de laboratorios para la confirmación molecular de influenza en todo el país. ${ }^{9}$ La crisis generada por esta emergencia sanitaria permitió la autorización de los recursos para construir nuevas y modernas instalaciones estratégicas. La mudanza y el traslado de materiales biológicos valiosos, incluyendo colecciones históricas de sustancias infecciosas, constituyeron un evento de seguridad nacional en términos de la gestión del riesgo biológico. ${ }^{10}$

En las ocho décadas de aportaciones a la salud pública, el Instituto se ha apoyado en la labor de su capital humano. A lo largo de su historia ha reunido (entre directivos, profesionales y personal técnico) a reconocidos expertos de la salud pública, un delegado permanente de la Organización de las Naciones Unidas para la Educación, la Ciencia y la Cultura y fundador del Colegio Nacional, delegados participantes de la constitución de la Organización Mundial de la Salud, un Premio Nacional de Ciencias, dos subsecretarios del ramo
Correspondencia: José Alberto Díaz-Quiñonez E-mail: alberto.diaz@salud.gob.mx
Fecha de recepción: 14-03-2019

Fecha de aceptación: 21-03-2019

DOI: 10.24875/GMM.19005161
Gac Med Mex. 2019;155:221-222

Disponible en PubMed www.gacetamedicademexico.com 
y una gran cantidad de científicos miembros del Sistema Nacional de Investigadores, de la Academia Mexicana de Ciencias, así como secretarios y presidentes de la Academia Nacional de Medicina de México, todos exponentes no solo de la ciencia y la técnica, sino del espíritu humanista. Lo anterior da cuenta de la preparación, el compromiso, el tamaño y el talento humano que ha ofrecido el Instituto a la salud pública.

En este contexto de consolidación de liderazgo, en el octogésimo aniversario de su fundación, el Instituto continúa su evolución. La propuesta de creación del Sistema Nacional de Diagnóstico en Salud, dependiente de la naciente Subsecretaría de Salud Pública, ${ }^{11}$ requerirá las atribuciones, la experiencia y la tradición del que, hasta hoy, ha sido el Instituto de Diagnóstico y Referencia Epidemiológicos "Dr. Manuel Martínez Báez". Para esta transformación será necesario considerar el motivo, el tiempo, el lugar, la persona, los medios y la forma. $\mathrm{Si}$ todo esto es reflexionado y ponderado de manera adecuada, a nuestro país le espera el éxito en términos de la política pública en salud, con un Instituto fortalecido y una firme visión de futuro.

\section{Bibliografía}

1. Paz O. El laberinto de la soledad, posdata y vuelta al laberinto de la soledad. México: Fondo de Cultura Económica; 2015.

2. Oficina Sanitaria Panamericana. Instituto Mexicano de Investigación. Bol Oficina Sanit Panam. 1938;269.

3. Martínez-Báez M. El Instituto de Salubridad y Enfermedades Tropicales. An Soc Mex Hist Ciencia y Tecnol. 1969;1:143-162.

4. Sepúlveda-Amor J. Editorial. Salud Publica México,1992;34:119-120.

5. Valdespino-Gómez JL, García-García ML, Del Río-Solezzi A, et al. El Instituto Nacional de Diagnóstico y Referencia Epidemiológicos "Dr. Manuel Martínez Báez" En: Una institución académica y dieciséis investigadores distinguidos. Quincuagésimo quinto aniversario del INDRE. México: Secretaría de Salud; 1994. p. 189-197.

6. Flisser-Steinbruch A, Velasco-Villa A, Martínez-Campos C, et al. Infectious diseases in Mexico. A survey from 1995-2000. Arch Med Res. 2002;33:343-350.

7. Flisser-Steinbruch A. Editorial. Salud Publica Mex. 2000;42:482-483.

8. Sarti E, Moreno-Galván M, Rodríguez-Angeles G, et al. Molecular characterization of anthrax in positive powders: a Mexican experience. J Clin Microbiol. 2003;41:4909.

9. Díaz-Quiñonez JA, Alpuche-Aranda CM. Métodos diagnósticos de influenza por laboratorio. En: Córdova-Villalobos, Valdespino-Gómez, Ponce-de-León. La epidemia de influenza A/H1N1 en México. México: Médica Panamericana; 2010. p. 87-105.

10. Ochoa-Carrera LA. Traslado de materiales biológicos valiosos a las nuevas instalaciones del Laboratorio Nacional de Referencia de México, Instituto de Diagnóstico y Referencia Epidemiológicos (InDRE). Tesis de Maestría en Gestión de la Salud, Universidad del Valle de México, Campus Lomas Verdes, 2014.

11. Academia Nacional de Medicina. La salud en la cuarta transformación de México. Sesión conjunta entre la Secretaría de Salud y la Academia Nacional de Medicina de México. Disponible en: https://m.youtube.com/ watch?v=Qp1JqOIHXGU\# 